\title{
GEOLOGISCHE VORAUSSETZUNGEN FÜR DAS WANDERN VON GEFÄLLSSTUFEN
}

\author{
HEINRICH JÄCKLI
}

Mit 1 Abbildung

In der geomorphologischen Literatur werden bestehende Gefällsstufen im Längenprofil von Flüssen oder von Tälern meist das Tal hinauf wandernd betrachtet. Das Aufwärtswandern solcher Steilstrecken wird dabei als Selbstverständlichkeit vorausgesetzt ${ }^{1}$, ohne daß immer untersucht würde, ob ein solches Wandern geologisch überhaupt möglich ist.

Im folgenden soll geprüft werden, unter welchen geologischen Voraussetzungen überhaupt ein Wandern von Gefällsstufen unter dem Einfluß fluviatiler Tiefenerosion in Betracht kommt. Was dabei für ein ganzes Tal gesagt wird, gilt sinngemäß auch für das einzelne Flußbett und umgekehrt.

\section{HÄRTESTÜFEN}

Härtestufen, ganz unabhängig von ihrer Größe, sind bedingt durch eine geologische Inhomogenität des Untergrundes, wobei der die Stufe verursachende Gesteinskomplexe relativ erosionsresistenter ist als seine Umgebung. Diese relative Erosionsresistenz braucht übrigens nicht in allen Fällen petrographisch bedingt zu sein, also im Mineralbestand, in der Struktur und der Textur des Gesteins zu liegen, sondern kann auch durch die Lagerung oder durch Richtung und Intensität der Gesteinsklüftung verursacht werden.

Eine solche Härteschwelle bildet eine lokale Erosionsbasis für das darüberfolgende Talstück oder die darüber folgende Flußstrecke.

Unterschiede in der Härte (genauer in der Erosionsresistenz) bedingen zur Hauptsache die relative Steilheit der Stufen, verglichen mit dem darüber und darunter angrenzenden Talabschnitt; im Flußlauf können bei günstigen Lagerungsverhältnissen die freistürzenden Wasserfälle entstehen, von denen der Niagara das Schulbeispiel ist.

Die Dicke, die Mächtigkeit des harten Komplexes, bedingt die Höhe der Stufe, soweit es sich um schichtförmige Lagerung handelt. Ist diese Dicke nicht konstant, so ändert mit dem Wandern der Stufe auch deren Höhe; wo die harte Schicht in der Richtung der Stufenwanderung auskeilt, verschwindet auch die Stufe.

Die Lage der Grenzfläche des erosionsresistenten Gesteinskomplexes bedingt die Richtung des Wanderns der Stufe. Dabei können folgende Fälle voneinander unterschieden werden (siehe Abbildung):

1. Die Grenzen stehen senkrecht.

Beispiel: Tessin bei Stalvedro $1 \mathrm{~km}$ östlich Airolo; steilstehende Gneise der LucomagnoDecke, im N von Rauhwacke mit Gips der Trias, im S von Bündnerschiefern begrenzt.

Die Stufe hat keine Möglichkeit zu wandern. Sie bleibt an Ort und Stelle stehen, wo sie erstmals entstanden ist, bis das Längenprofil ausgeglichen und die Erosionsterminante erreicht ist.

2 a. Die Grenzen fallen talabwärts mit kleinerem Winkel als die Talsohle.

Beispiel: Fundogn Vallatscha, Ostende von Alp Anarosa, Gemeinde Wergenstein, Schams, Graubünden; hartes, kalkiges „Hauptkonglomerat " des Flysches über weicheren Tonschiefern fällt mit $10^{\circ}$ nach SW; Stufenhöhe ca. $250 \mathrm{~m}$.

\section{b. Die Grenzen liegen horizontal.}

Beispiel : Fistelbach $1 \mathrm{~km}$ SE Fischental, Kt. Zürich; mächtige Nagelfluhschicht horizontal gelagert, in Wechsellagerung mit weicheren Mergeln und Sandsteinen der Molasse.

Als analoge Beispiele ungezählte Stufen in der Schichtstufenlandschaft der horizontalen Molasse, besonders des Napf- und Hörnlischuttfächers.

1 O. MAULL: Geomorphologie. Leipzig-Wien, 1938. 


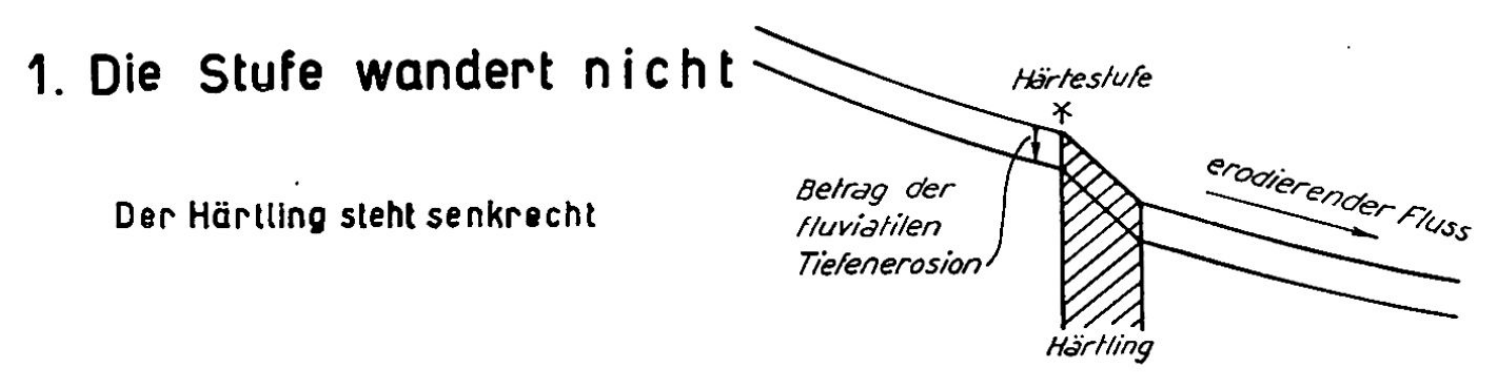

\title{
2. Die Stufe wandert auf wärts
}

a. Der Härtling fällt talabwärts mit kleinerem Winkel als die Talsohle

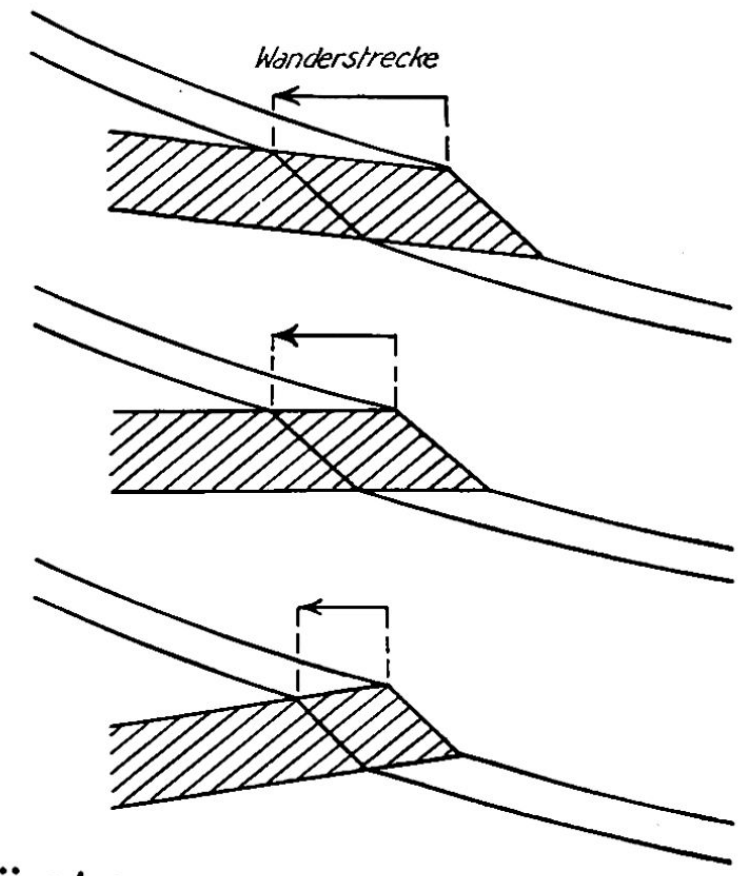

\section{Die Stufe wandert a bwärts}

c. Der Härtling fällt talaufwärts

\begin{abstract}
Der Härtling fällt talabwärts mit grösserem Winkel als die Talsohle
\end{abstract}

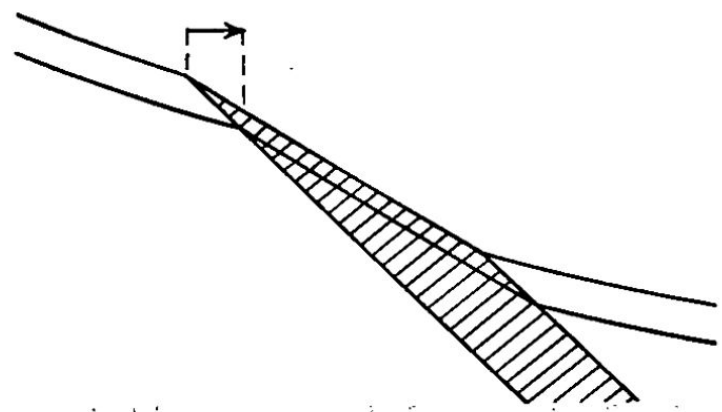

Das Wandern von Härtestufen bei fluvialer 'Tiefenerosion

c. Die Grenzen fallen talaufwärts.

Beispiel: Thur zwischen Starkenbach und Stein im Toggenburg; Durchbruch durch die Alpenrandkette; untere Kreide auf Flysch und Molasse, nach SE einfallend.

In allen drei Fällen 2a, 2b und $2 \mathrm{c}$ wandert die. Härtestufe talaufwärts. Dieser Vorgang kann sich in den Fällen 2a und $2 b$ über unbegrenzte Distanzen vollziehen, solange der stufenbildende Härtling vorhanden ist. Die Stufe kann sich dabei erhöhen oder erniedrigen, je nach den Mächtigkeitsschwankungen der stufenbildenden harten Schicht. Sie kann sich aber auch versteilen und im Flußbett zum freien Überfall werden oder verflachen, wenn es wechselnde Härteunterschiede zwischen Liegendem und Hangendem gestatten. 
3. Die Grenzen fallen talabwärts mit größerem Winkel als die Talsohle.

Beispiel: Hinterrhein in der Rofla zwischen Sufers und Andeer (Graubünden); Granitgneis der Suretta-Decke nach E einfallend, darunter weiche Bündnerschiefer der Splügenermulde.

In diesem Fall wandert die Stufe talabwärts, und zwar mit den unter Ziffer 2 gegebenen Charakteristiken, allerdings mit der Ausnahme, daß dabei keine größeren Distanzen zurückgelegt werden können.

4. Unter- und Obergrenze verlaufen nicht parallel, sondern fallen in umgekehrter Richtung, die eine talaufivärts, die andere talabwärts; Ober- und Untergrenze der dadurch bedingten Stufen wandern ebenfalls in umgekehrter Richtung.

a. Divergieren die Gesteinsgrenzen nach unten, so entfernen sich die beiden Stufenenden voneinander, die Stufe wird immer länger.

Beispiel : Antiklinalklusen des Juragebirges.

b. Konvergieren die Gesteinsgrenzen nach unten, so bewegen sich die Stufenenden zueinander hin, die Stufe wird damit immer kürzer und verschwindet bei ciner muldenförmigen Schichtumbiegung mit der Zeit ganz.

Beispiel : Sernf zwischen Schwanden und Engi, Kt. Glarus; Verrucano auf Flysch.

\section{ANDERE STUFEN, „NICHTHÄRTESTUFEN“}

Kommen in der Talsohle junge Bergsturzbildungen, Fels- oder Schuttrutschungen aus den Talflanken, Schuttkegel von Seitenbächen oder Moränenwälle zur Ablagerung, so entsteht an deren talabwärtigem Rand eine Gefällsvergrößerung, talaufwärts ungekehrt eine Gefällsverminderung, meist kombiniert mit flachen Flußalluvionen, sodaß gesamthaft im Längenprofil eine sog. "Akkumulationsstufe » rorliegt.

Hat der Fluß die stufenverursachende Akkumulation durchsägt, so besitzt er wieder sein früheres ausgeglichenes Längenprofil, ohne daß die einstige Stufe irgend eine Möglichkeit gehabt hätte, weiter talaufwärts zu wandern als bis zum oberen Ende des stufenbildenden Hindernisses.

Beispiel: Vorderrhein im Flimserbergsturz zwischen Reichenau und Ilanz.

Bei andern Nichthärtestufen kann es sich beispielsweise um tektonische Stufen. um Epigenesen oder glaziale Stufen handeln, jedenfalls um Stufen im Fels, wobei für die folgenden Überlegungen immer homogener Üntergrund vorausgesetzt wird.

OtTo LeHMANN ${ }^{2}$ hat überzeugend dargetan, daß für solche Stufen ein Aufwärtswandern über große Distanzen aus hydraulischen Gründen ausgeschlossen ist. Der Fluß hat die Tendenz, ein ausgeglichenes Längenprofil, die sog. Erosionsterminante, zu erreichen. Die Stufe muß unter dem Einfluß fluviatiler Erosion abflachen. $\mathrm{lhr}$ oberes Ende wandert rascher flußaufwärts als ihr unteres Ende, sodaß aus dieser Verlängerung der ganzen Stufe eine Verflachung resultieren muß, bis schließlich überhaupt nicht mehr von einer Stufe gesprochen werden kann.

In keincm Fall kann die «Nichthärtestufe» durch fluviatile Bearbeitung stei- ler werden, wie das bei der Härtestufe möglich ist. Sie kann zudem nur aufwärts wandern, aber nie stille stehen oder gar abwärts wandern wie die Härtestufe.

\section{SAMMELSTUFEN}

Eine relative sehr resistente Härtestufe kann eine tiefere, verglichen mit ihr etwas weichere und damit rascher wandernde. Härtestufe aufhalten und in sich vereinigen; sie wird dann nach A. Annaheim zur sog. "Sammelstufe » ${ }^{3}$. Auch Nicht-

2 Otro LehmanN: Gefällsentwicklung und Talstufen im Hochgebirge. Zsch. f. Geomorphologie, Bd. X, 1937. 
härtestufen können sich mit einer Härtestufe zu einer Sammelstufe vereinigen, die dann auch den Regeln einer reinen Härtestufe wandern muß.

Zusammenfassend stellen wir fest, daß ein Wandern von Härtestufen, selbst über größere Distanzen, nicht schlechthin verneint werden darf, daß es anderseits aber auch nicht angeht, bei jeder Stufe ein solches Wandern a priori vorauszusetzen. Vielmehr sind, wie stets in der genetisch orientierten Morphologie, die petrographischen und tektonischen Verhältnisse für jeden einzelnen Fall zu prüfen.

\section{CONDITIONS GÉOLOGIQUES DU DÉPLACEMENT DES GRADINS DE CHUTE}

Si un fleuve en plein travail d’érosion coule sur un sol de dureté variée, il en résulte dans son profil en long ce qu'on appelle des gradins de chute. L'article montre quelles sont les conditions tectoniques pour que ces gradins de dureté se déplacent en amont ou en aval ou restent stationnaires. Un graphique rend cela plus clair. Des gradins qui sont dus à des éboulements récents ou à des cônes de déjection latéraux ne peuvent se déplacer que jusqu'à l'extrémité supérieure de l'obstacle; au-delà le profil en long est de nouveau régularisé. Des gradins résultant de mouvements de la croûte terrestre, de l'épigénèse ou de l'érosion glaciaire peuvent bien remonter le fleuve, mais perdent peu à peu de leur hauteur et finissent par disparaitre.

\section{LE PREMESSE GEOLOGICHE PER LA MIGRAZIONE DI UNA SOGLIA ROCCIOSA}

Se il substrato dell'alveo di un fiume, in efficienza erosiva, presenta rocce di diversa durezza, lungo il profilo longitudimale della valle, si formano le cosiddette "soglie dure “. Il presente lavoro dimostra quali siano le premesse tettoniche perchè una soglia possa migrare verso il basso, o verso l'alto della valle, o restare immutata sul posto: il risultato è illustrato in una figura. Soglie create da franamenti o da coni di dejezione di torrenti laterali possono migrare solo fino al limite superiore dello sbarramento dove il profilo ritorna uguagliato. Soglie, generate da movimenti tettonici della crosta terrestre, da epigenesi e da erosione glaciale, possono migrare verso monte, ma diminuiscono di sbalzo fino all'appianamento completo.

\section{DER 17. INTERNATIONALE GEOGRAPHENKONGRESS IN WASHINGTON}

\section{ERICH Schwabe}

Die Bundeshauptstadt der Vereinigten Staaten war anläßlich der Zusammenkunft in Lissabon vor drei Jahren dazu ausersehen worden, im Sommer 1952 den 17. internationalen Geographenkongreß, den zweiten seit Kriegsende, zu beherbergen. Die Versammlung, zu der sich im August über tausend Teilnehmer aus aller Welt, rund zwei Drittel davon aus den U.S. A. selber, einfanden, war ausgezeichnet vorbereitet, was ebensosehr die wissenschaftliche Arbeit, wie den nicht minder wertvollen persönlichen Kontakt unter den Anwesenden erleichterte.

Den eigentlichen Anlaß, die Veranstaltung nach fast einem halben Jahrhundert einmal wieder in den U.S.A. abzuhalten, gab die Feier des 100-jährigen Bestehens der American Geographical Society. Diese Gesellschaft, die in New York ihren Sitz hat, teilt sich mit einer Schwesterorganisation, der. National Geographic Society in Washington, in die Aufgabe, der Geographie im Volke weiten Widerhall $\mathrm{zu}$ verschaffen und ihr durch wissenschaftliche Untersuchungen, Veröffentlichungen und Expeditionen Gewicht zu verleihen. War ihr Bestreben zu Beginn ihrer Tätigkeit vor allem dahin ausgerichtet, die Kenntnisse über Neuentdeckungen in unerforschten Ländern zu mehren - man denke an Innerafrika, an weite Gebiete Südamerikas und Australiens, die damals, vor 100 Jahren, noch vollkommene Terra incognita waren -, so ging sie später mehr und mehr zur Vertiefung des Wissens, zur Analyse des in den Hauptzügen nun bekannten Oberflächenbildes der Erde über. Mit Publikationen, nicht zuletzt mit bedeutsamen Kartenerscheinungen, mit einer überaus reich dotierten Bibliothek stellt sie sich der Öffentlichkeit zur Verfügung; ihre Dienste werden neuer-

${ }^{8}$ H. Annaheim: Studien zur Morphogenese der Südalpen zwischen St. Gotthard und Alpenrand. Geographica Helvetica 1, 1946, Heft 2. 